\title{
DISEÑO DE UN SISTEMA DE GESTIÓN EN RESPONSABILIDAD SOCIAL EMPRESARIAL PARA PEQUEÑOS HOTELES DE LA CIUDAD DE CARTAGENA*
}

\author{
José Julio Vergara Arrieta ${ }^{1}$ \\ Adolfo Enrique Carbal Herrera ${ }^{2}$
}

\begin{abstract}
RESUMEN
El presente trabajo de investigación corresponde al diseño de un sistema de gestión en responsabilidad social empresarial para pequeños hoteles de la ciudad de Cartagena, con base en la caracterización y evaluación de sus prácticas en este campo, pretendiendo aportar una poderosa herramienta para guiar los procesos de implementación de acciones o actividades de carácter socio-ambiental en estas unidades empresariales. El turismo es considerado como una de las apuestas productivas trascendentales del departamento de Bolívar, por su dinamismo, oferta de empleos, redistribución de la riqueza y contribución al desarrollo regional. Este sector mueve gran parte de la economía de la ciudad de Cartagena y del mismo dependen muchos de sus pobladores, ya sea directa o indirectamente. Sin embargo, el turismo de la ciudad presenta diversas problemáticas que afectan su competitividad y sustentabilidad, como procesos de gestión inadecuados y la baja disponibilidad de personal calificado. En este sentido la responsabilidad social empresarial, juega un papel muy importante, en la medida que sea entendida como una herramienta de gestión y compromiso al que se adhieren las unidades empresariales, permitiéndoles mejorar el dinamismo de sus operaciones y orientar sus procesos internos de tal manera que se puedan alcanzar niveles idóneos de bienestar de forma integral en toda la organización. Para el desarrollo de la investigación se realizó una extensa exploración de información sobre la temática combinado con un trabajo de campo en pequeños hoteles de la ciudad, para finalmente, y después del análisis de datos documentales y aquellos extraídos directamente de la realidad objeto de estudio, proceder al diseño del sistema de gestión para pequeños hoteles de la ciudad de Cartagena.
\end{abstract}

\section{PALABRAS CLAVES}

Pequeños hoteles, responsabilidad social empresarial, sistemas de gestión, turismo.

\begin{abstract}
This research paper corresponds to the design of a management system in social corporal responsibility for small hotels in Cartagena, based on the characterization and evaluation of its practices in this field, it provides a powerful tool to guide the processes of implementation of actions or activities of socio-environmental nature in these corporate units. Tourism is considered as one transcendental production betting in Bolivar due to its dynamism, job offering, wealth distribution and contribution to the regional development. This sector moves a great deal of the economy in Cartagena and many of the inhabitants depend on it directly or indirectly. However, tourism in Cartagena presents different issues that affect competitiveness and sustainability as inadequate management processes and the low availability of qualifies staff. In this sense,
\end{abstract}

Depositado en agosto 15 de 2014, aprobado en octubre 31 de 2014.

*El presente artículo es resultado del proyecto de investigación titulado Diseño de un sistema de gestión en Responsabilidad Social Empresarial para PYMES del sector turismo: Caso pequeños hoteles de la ciudad de Cartagena.

1 Magister (c) en Administración de la Universidad de Medellín, Especialista en Finanzas y Contador Público de la Universidad de Cartagena. Docente investigador en Contabilidad Social y Ambiental de la Universidad de San Buenaventura. Miembro del Grupo de Investigación en Gestión Administrativa y Contable-GICAC. Email: jovear6@hotmail.com

2 Contador Público, Magister en ciencias ambientales del SUE Caribe. Docente investigador de la Universidad de Cartagena y de la Universidad Libre, Sede Cartagena. Director del grupo de investigación GIDEA de la Universidad de Cartagena e Integrante del grupo de investigación GISEMA de la Universidad Libre, Sede Cartagena. E-mail: carbal125@yahoo.es 


\begin{abstract}
the corporate social responsibility plays an important role as long as it is understood as a management and commitment tool to which the corporate units adhere, allowing improvement in the dynamism of its operations and orientation of its internal processes in such a fashion that they can reach optimum levels of welfare in all the organization.

For the development of this research we explored information on this topic along with fieldwork in small hotels in the city, and finally, after documentary data analysis, plus those taken directly from the reality of our subject of study, we continued to design the management system for small hotels in Cartagena.
\end{abstract}

\title{
KEYWORDS
}

Small Hotels, Corporal Social Responsibility, Management Systems, Tourism.

\section{INTRODUCCIÓN}

La responsabilidad social empresarial se ha convertido en uno de los temas que circunda los ambientes empresariales y académicos que pretenden dar nuevos giros al actuar organizacional. Esta situación se ha visto reforzada en la medida que otros enfoques y elementos como el desarrollo sostenible, el balance social y el desarrollo de la ética empresarial también han propiciado la creación de espacios para discutir y crear nuevas formas de pensamiento fundados en una gestión responsable con la sociedad y el medio ambiente. La situación descrita también se origina en la presión que se ejerce desde las esferas sociales para la consecución de una sociedad más justa y con condiciones ambientales idóneas.

Si bien el Sistema de Gestión en Responsabilidad Social Empresarial para Pequeños hoteles de la Ciudad de Cartagena se estructuró atendiendo diversos modelos a nivel mundial que buscan gestionar eficientemente la responsabilidad social empresarial, es necesaria la contextualización de estas herramientas a las características propias de la realidad doméstica, para que así se puedan generar políticas y prácticas que armonizadas con las circunstancias que se viven en estas unidades empresariales puedan generar valor agregado y diferenciador con respecto a organizaciones similares de otros destinos turísticos.

El Sistema de Gestión en Responsabilidad Social Empresarial para Pequeños hoteles de la Ciudad de Cartagena permite a estas unidades empresariales gozar de una orientación simple, efectiva y acorde con su realidad, dado los obstáculos que presentan las pequeñas em- presas, algunos de orden económico y otros estructurales, para certificarse en normas internacionales como la SA 8000, o para implementar guías de responsabilidad social como la ISO 26000 y la misma guía colombiana GTC 180 del ICONTEC. Por ello la trascendencia de dicha herramienta, la cual no implica mayores costos para estos entes y que está diseñada puntualmente para atender las particularidades e implicaciones de estas organizaciones y la relación con sus stakeholders. En otras palabras, la herramienta construida se contextualiza a las particularidades de las entidades objeto de análisis, sin desconocer las generalidades inherentes a la temática.

Este artículo es resultado del proyecto de investigación titulado: diseño de un sistema de gestión en responsabilidad social empresarial para pequeños hoteles de la ciudad de Cartagena. El cual partió de la caracterización de los pequeños hoteles de la ciudad, para finalmente proceder al diseño del modelo de gestión social, armonizado a las particulares propias de la población objeto de estudio, el cual se expone en este documento.

\section{MARCO REFERENCIAL}

Numerosos estudios como los realizados por Castellanos (2003), Sierra y Londoño (2008), y el Departamento Nacional de Planeación DNP (2007) señalan la importancia que tienen las micro, pequeñas y medianas empresas (de ahora en adelante MiPymes) para la economía del país. Según el Departamento Administrativo Nacional de Estadística - DANE, las microempresas constituyen el $96,4 \%$ de las empresas en Colombia, el 3,0\% las pequeñas, el 
$0,5 \%$ las medianas y solo el $0,1 \%$ las grandes (Carbal, 2009).

De acuerdo con el Censo de 2005, la estructura empresarial colombiana está principalmente conformada de Pymes, las cuales constituyen la principal fuente de empleo en Colombia. Un alto porcentaje de este tipo de organizaciones se dedica a una gran variedad de actividades del sector servicios $(59,2 \%)$. El comercio y la industria le siguen en importancia similar con 21,5 y 19,3\% respectivamente (Sánchez, Juliao y Zuluaga, 2013; Montoya, Montoya y Castellanos, 2010; Garzón, 2005; Giraldo, 2007).

Las MiPymes son actores estratégicos en la creación de empleo, esencialmente para individuos poco calificados que frecuentemente encuentran barreras para entrar a laborar en empresas más grandes y con mayores exigencias (DNP, 2007). También se constituyen en ejes formadores de bienestar social, dado que contribuyen al sostenimiento de miles de familias, y a la transformación de la industria nacional (Supersociedades, 2005).

A pesar de su papel relevante dentro de la economía doméstica, estas unidades empresariales presentan serias falencias que constantemente ponen en riesgo su permanencia en el tiempo. Estas presentan problemáticas como: baja capacidad de innovación, bajo uso de tecnologías de información y comunicaciones, limitado acceso a financiamiento adecuado, escasa cultura financiera, ausencia de sistemas de información gerencial, pocas estadísticas a cerca de sus procesos productivos, poca competitividad, problemas para la comercialización de sus productos y la obtención de insumos y la limitada participación en el mercado de la contratación pública (Zevallos, 2006; Castellanos, 2003; Velásquez, 2004; Sánchez, Osorio y Baena, 2007; Sánchez, Juliao y Zuluaga, 2013; Rodríguez, 2003; Montoya, Montoya y Castellanos, 2010; Melo, 2003; Puyana, 2010).

Por otra parte, las empresas inmersas dentro del sector turismo de Cartagena tienen gran responsabilidad en el dinamismo de la ciudad y el desarrollo de su economía. Cartagena se ha consolidado como un destino turístico por excelencia, dado su tradicional arquitectura colonial y su estatus de Patrimonio Cultural de la Humanidad (Mendoza, 2012; Quintero, Bernal y López, 2005; Alcaldía Mayor de Cartagena, 2004; Sierra, Marrugo y Quejada, 2004). Como ciudad turística, Cartagena recibe visitantes de diferentes índoles, que necesitan servicios de alojamiento, alimentación y esparcimiento de calidad y confort. Es por ello, que nace la necesidad de fortalecer empresas que prestan servicios turísticos como hoteles, para que la ciudad pueda seguir en la línea del mejoramiento de su capacidad competitiva (Maldonado, 2006; Ramos, 2012; Otero, 2008).

Los pequeños hoteles de Cartagena no escapan a la problemática planteada, por lo cual se constituyeron en la población objeto de estudio, en concordancia con la importancia que tienen dentro de la economía de la ciudad. Según el Plan Regional de Competitividad Cartagena y Bolívar 2008-2032, publicado en mayo de 2010 por la Comisión Regional de Competitividad de Cartagena y Bolívar, el departamento y principalmente la ciudad amurallada enfrentan las siguientes debilidades referentes al sector turismo:

- Altos niveles de informalidad.

- Altos costos y acceso restringido a los servicios públicos.

- Baja disponibilidad de recurso humano calificado, especializado y bilingüe, especialmente en las actividades económicas asociadas a las apuestas productivas.

- Escasa participación de los actores importantes involucrados en las iniciativas de competitividad.

- Debilidad en la información turística.

- Sistema tributario oneroso, inestable y complejo.

Ante la problemática expuesta sobre las MiPymes, una de las alternativas que posibilita contrarrestar la misma, reside en la construcción de sistemas de gestión a su interior para propiciar su desarrollo integral, como es posible a través de la implementación de prácticas de responsabilidad social empresarial. Un sistema de gestión es una estructura probada para la actividad y mejoramiento continuo de las políticas, los procedimientos y procesos de la organiza- 
ción; permite crear una arquitectura de gestión que sustente la interrelación entre las políticas, estrategias, comportamientos y procesos de la empresa, y consolidar el comportamiento ético (Ogalla, 2006; Gómez, 2009; Pinto, Becerra y Gómez, 2012; Solana y Pérez, 2008).

Según el Instituto Ethos de Empresas y Responsabilidad Social y el Serviço Brasileiro de Apóioás Micro e Pequeñas Empresas - SEBRAE (2007)

\section{"La Responsabilidad Social Empresarial es la forma de gestión que se define por la relación ética y transparente de la em- presa con todos los públicos con los cua- les se relaciona y por el establecimiento de metas empresariales compatibles con el desarrollo sostenible de la sociedad, preservando recursos ambientales y cul- turales para generaciones futuras, respe- tando la diversidad y promoviendo la re- ducción de las desigualdades sociales".}

Según la GTC 180 de la Responsabilidad Social (ICONTEC, 2008):

La responsabilidad social empresarial es el compromiso voluntario y explícito que las organizaciones asumen frente a las expectativas y acciones concertadas que se generan con las partes interesadas (stakeholders), en materia de desarrollo humano integral; ésta permite a las organizaciones asegurar el crecimiento económico, el desarrollo social y el equilibrio ambiental, partiendo del cumplimiento de las disposiciones legales.

Según la guía ISO 26.000 (citado por Veloz, 2008) la Responsabilidad Social se concibe como

la responsabilidad de una organización ante los impactos que sus decisiones y actividades ocasionan en la sociedad y el medio ambiente, a través de un comportamiento transparente y ético que:
- Contribuya al desarrollo sostenible, incluyendo la salud y el bienestar de la sociedad;

- Tome en consideración las expectativas de sus partes interesadas (stakeholders);

- Cumpla con la legislación aplicable y sea coherente con la normativa internacional de comportamiento; $y$

- Esté integrada en toda la organización y se lleve a la práctica en sus relaciones.

Un sistema permite integrar la Responsabilidad Social Empresarial en la estrategia de la organización con información sistemática y comunicación efectiva, a través de un liderazgo responsable, con un lineamiento estratégico y desplegado en los diferentes grupos de interés ${ }^{3}$ o stakeholders, orientado a tener una gestión de relaciones e involucramiento sistemático con estos grupos de interés prioritarios, logrando resultados e indicadores que pueden ser monitoreados periódicamente para obtener en forma gradual las mejores prácticas de responsabilidad social (Guajardo, 2007; Del Castillo, Bonnet, Hennessey, Quiroz y Rizzo, 2012).

Un sistema de gestión en responsabilidad social brinda a las organizaciones el incremento en su competitividad, mejoras en el proceso de la toma de decisiones al entender las expectativas de sus grupos de interés, mayor confianza y reputación ante la sociedad y competidores, la lealtad y moral de los trabajadores se incrementa, aumento en su capacidad de innovación al acceder a perspectivas de los grupos de interés, y principalmente, ahorros para la organización por mejoras en la productividad, uso eficiente de recursos, menor consumo de agua y energía, reducción del desperdicio y recuperación de materiales (Molina, 2009; Mora, 2005; Aristimuño, Velásquez y Rodríguez, 2010).

Igualmente el sistema de gestión en Responsabilidad Social Empresarial beneficia a las organizaciones en la medida que permite una diferenciación de los competidores, permite la

\footnotetext{
3 El Global Reporting Iniciative - GRI (2006) define como grupos de interés "aquellas entidades o individuos a los que pueden afectar de manera significativa las actividades, productos y/o servicios de la organización; y cuyas acciones pueden afectar dentro de lo razonable a la capacidad de la organización para desarrollar con éxito sus estrategias y alcanzar sus objetivos".
} 
captación de nuevos clientes y mantener los actuales, mejora las condiciones laborales y aumenta la moral de los trabajadores, mejora la reputación ante toda la sociedad y principalmente permite la obtención de información válida y suficiente para demostrar transparencia ante las parte interesadas (stakeholders), que de otro modo no se podría obtener (Fontalvo y Bolívar, 2010).

La responsabilidad social entra a formar parte de la estrategia organizacional de aquellos entes que asumen compromisos coherentes con la realidad mundial (Valverde y Bermúdez, 2012), donde los escándalos corporativos han dejado en entredicho la reputación del sector empresarial.

\section{METODOLOGÍA}

La presente investigación es de naturaleza descriptiva, ya que el objeto de la misma permitió establecer una caracterización de la realidad de los pequeños hoteles de la ciudad de Cartagena. Para el logro del objetivo trazado se llevaron a cabo las siguientes fases o etapas:

Fase 1. Identificación de la población objeto de estudio y determinación de la muestra. La población objeto de estudio está compuesta por Pequeños hoteles de la ciudad de Cartagena registrados formalmente, ubicados en el centro histórico y en el barrio de Bocagrande, cuyo número de habitaciones no supera veinticinco (25). Para la selección de la muestra se siguió un procedimiento intencional, que consistió en contactar el mayor número de pequeños hoteles de ciudad, y aquellos que demostraron accesibilidad constituyeron la muestra. A través de cartas formales, llamadas telefónicas y correos electrónicos se contactaron sesenta (60) pequeños hoteles de la ciudad, de los cuales el 33\% expresaron su interés y disponibilidad para participar en la investigación.

Fase 2. Caracterización preliminar. Los hoteles que constituyeron la muestra se caracterizaron mediante un proceso de observación e indagación preliminar, cuyos resultados fueron consignados en diarios de campo y planillas de observación.
Fase 3. Diseño y aplicación de cuestionarios. Se procedió a la construcción de instrumentos de recolección de información (cuestionarios), con base en modelos de indicadores de diversos organismos e instituciones académicas a nivel nacional e internacional, y tomando como referente contextual la problemática socioambiental detectada en la ciudad de Cartagena. Luego estos fueron aplicados a gerentes y grupos de interés de las organizaciones objeto de análisis, quienes previamente fueron identificados $\mathrm{y}$ caracterizados.

Fase 4. Estudio empírico (seguimiento de casos). Se efectuaron estudios de casos que permitieron la contrastación de la información recolectada mediante los instrumentos aplicados y la realidad observada. Este proceso se llevó a cabo en ocho (8) pequeños hoteles, mediante visitas programadas a dichos establecimientos. Se realizó un proceso de observación-interacción, dado que los elementos de interés que se consignaron en los instrumentos fueron analizados en la realidad (observación) y algunos elementos se estudiaron a través de la interacción con distintos stakeholders. Los registros se consignaron en una lista de chequeo.

Fase 5. Procesamiento y análisis de la información. La información que se obtuvo mediante la aplicación de las encuestas se procesó a través de la aplicación Microsoft Excel y después se cruzó, con la información obtenida mediante el proceso de observación-interacción, para realizar los análisis del caso.

Fase 6. Diseño y presentación del sistema de gestión social. Finalmente se procedió a la construcción del Sistema de Gestión Social para las organizaciones objeto de estudio. Como se mencionó anteriormente, se tomaron diversos modelos a nivel mundial, con base en los cuales se construyó uno integral y armonizado a la realidad de los pequeños hoteles participantes.

\section{RESULTADOS}

Los hoteles que participaron en esta investigación cumplen con todos los requisitos legales para el desarrollo de su objeto social y con to- 
das las obligaciones contractuales que emanan de la legislación laboral colombiana. Como se mencionó anteriormente, están ubicados en el Centro Histórico y el barrio Bocagrande de la ciudad de Cartagena de Indias, y sus huéspedes en su gran parte visitan la ciudad por motivos de esparcimiento, eventos de tipo académico y/o empresarial.

El 55\% de los hoteles participantes conocen el concepto de responsabilidad social empresarial y el $27 \%$ lo conocen en parte. Los análisis realizados evidencian que los pequeños hoteles de la ciudad implementan acciones de corte social fundadas en una gestión responsable, aunque por el desconocimiento de la teoría referente al tema, muchas veces no se percibe de esa manera. De la misma forma, la totalidad de los hoteles de la muestra no documentan sus procesos, por lo cual no se cuenta con información para la construcción de informes de corte social.

Por otra parte, se pudo constatar que la mayoría de los hoteles (67\%) difunde su misión, visión y metas institucionales entre sus empleados, teniéndolas visibles ante todos en la organización, pero los resultados del diagnóstico reflejan que un porcentaje igual no ha incorporado consideraciones sobre responsabilidad social empresarial en su misión, visión y metas institucionales. Todos estos aspectos buscan el desarrollo de estrategias diferenciadoras que puedan dar un valor agregado a los pequeños hoteles de Cartagena, en la consecución de nuevos segmentos de clientes, como el turismo corporativo, que ha aumentado en la ciudad y representa un renglón que atendido de manera eficiente puede dejar muchos beneficios para los hoteles de la ciudad. En razón de ello, el 64\% de los hoteles manifiesta que el sector del turismo corporativo puede traer grandes beneficios a la organización $\mathrm{y}$, más aun, el $73 \%$ expresa que tienen todas las condiciones para prestar un servicio de calidad y confort a este segmento.

Se cuenta con un manual de funciones y procedimientos que es dado a conocer a los empleados en el momento de su inducción a la empresa (82\%). Esto demuestra seriedad en la gestión del talento humano y los esfuerzos por incentivar una cultura organizacional de me- jora continua y de especialización de las actividades de cada empleado. En la parte que se encuentran rezagados es en la construcción de un código de ética y/o conducta que integre todos los procesos organizacionales con fundamentos éticos y morales, aunque es preciso recalcar que dentro de los manuales de funciones y procedimientos se incorporaron algunas consideraciones al respecto.

Por otro lado, en aspectos medioambientales se evidenciaron algunos elementos sobresalientes, como el uso de mecanismos para minimizar los impactos causados al medio ambiente, entre los que se destacan las bombillas ahorradoras y filtros para el ahorro del agua. Asimismo, se destaca el empuje que ha dado la administración a procesos que buscan el uso racional del agua, energía y el gas natural para aquellos hoteles que incluyen servicio de comedor para sus huéspedes, y la disminución en la producción de residuos sólidos. Asimismo, el 55\% de los hoteles recicla, aunque el resto manifiesta que no lo hacen debido a los procesos tradicionales que emplean las empresas encargadas de recolectar las basuras de la ciudad, donde a pesar de que exista una separación en la fuente de los residuos, estos se mezclan en el camión recolector.

Los puntos que implican mejoras en la gestión ambiental de los pequeños hoteles de Cartagena son los siguientes: Solo el 27\% de los hoteles de la muestra cuenta con una política medioambiental definida y conocida por todos los empleados y ninguno brinda capacitaciones a sus trabajadores en este tema. Igualmente, el 55\% no cuenta con procesos que permitan prever los impactos de la utilización de determinados productos en el medio.

\section{SISTEMA DE GESTIÓN EN RESPON- SABILIDAD SOCIAL EMPRESARIAL PARA PEQUEÑOS HOTELES DE LA CIUDAD DE CARTAGENA}

La RSE es de voluntaria implementación, ésta nace del deseo, el cual posteriormente se transforma en un compromiso de los empresarios que tienen la certeza que la gestión social responsable se constituye en una estrategia para mejorar y adquirir matices diferenciadoras que 
crean y dan ventajas competitivas. Por ello, el empresario que desee implementar este sistema de gestión social adquiere un compromiso con la sociedad y consigo mismo, dado que se compromete a desarrollar políticas sociales acordes con la realidad del sector y a mejorar su gestión interna.

El Sistema de Gestión en Responsabilidad Social Empresarial para Pequeños hoteles de la Ciudad de Cartagena se constituye en una herramienta que proporciona un direccionamiento para realizar una gestión responsable en estas unidades empresariales. Para su diseño se han tomado como referencia diversas normas, estándares, principios, prácticas y modelos en responsabilidad social empresarial de todo el mundo, principalmente de Latinoamérica, y la interpretación que se hizo de la realidad actual de las prácticas sociales y ambientales de los pequeños hoteles de la ciudad de Cartagena. Este sistema tiene como fin fa- cilitar la integración de principios y prácticas sociales y ambientales en los pequeños hoteles de la ciudad de Cartagena, proporcionando a estas organizaciones una estructura coherente con su realidad, que se integre en sus procesos organizacionales, de tal manera que éstos puedan ejecutarse bajo lineamientos responsables, y sus impactos sobre la sociedad y el medio ambiente sean minimizados.

\subsection{ETAPAS DEL SISTEMA}

El Sistema de Gestión en Responsabilidad Social Empresarial para Pequeños hoteles de la Ciudad de Cartagena cuenta con cuatro (4) etapas plenamente interrelacionadas que conforman un ciclo de gestión, cada una con un propósito específico que guía fácilmente las acciones a seguir en un direccionamiento estratégico y socialmente responsable. Del mismo modo, se constituye en un proceso sistemático de mejora continua. Las etapas son las siguientes:

\section{Gráfica 1. Sistema de Gestión en Responsabilidad Social Empresarial para Pequeños hoteles de la Ciudad de Cartagena}

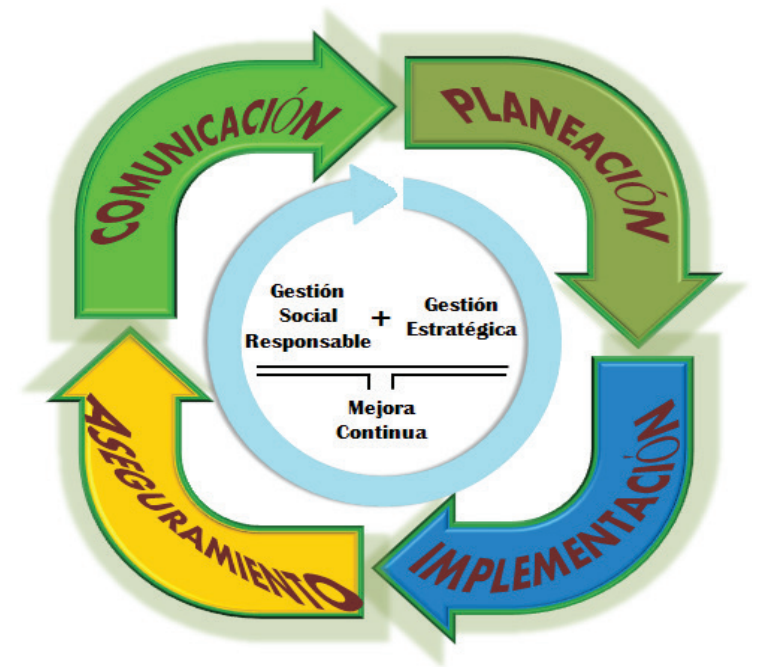

Fuente: elaboración propia

\subsubsection{Planeación}

Esta etapa se constituye como el punto de partida para la implementación de estrategias socialmente responsables dentro de las organizaciones. Se establece como la estructura que orientará todos los procesos y actividades del Sistema de Gestión en Responsabilidad Social
Empresarial para Pequeños hoteles de la Ciudad de Cartagena. Su importancia radica en el principio de que una buena planeación permite la obtención de los resultados deseados. Esta etapa se nutre del análisis elaborado a las prácticas realizadas por la organización en materia social y ambiental. 
Una correcta planeación de un enfoque de responsabilidad social empresarial se estructura con las siguientes fases:

\subsubsection{Identificación de Grupos de Interés} La teoría de la responsabilidad social empresarial expone que los Grupos de Interés de cualquier organización empresarial son principalmente sus empleados y/o colaboradores, sus clientes, sus proveedores, la comunidad de influencia, sus socios y/o accionistas y el Estado. Algunos teóricos van mucho más allá de estos, al incluir a la competencia y toda la sociedad. Para el Sistema de Gestión en Responsabilidad Social Empresarial para Pequeños hoteles de la Ciudad de Cartagena se han definido cuatro (4) stakeholders, los cuales comprenden los grupos más cercanos e importantes dentro de una gestión integral en organizaciones del sector: Empleados, clientes, proveedores y comunidad de influencia. Se pretende crear un listado agrupando a las personas, grupos, instituciones $\mathrm{u}$ organizaciones que pertenezcan a cada uno de estos grupos de interés. A continuación se exponen algunas consideraciones a tener en cuenta para la identificación de los stakeholders.

\subsection{Empleados}

Los empleados son los stakeholders más cercanos y sensibles de cualquier organización. Al mismo tiempo son considerados como los más importantes dentro de las empresas que constituyen el sector objeto de estudio (pequeños hoteles de la ciudad de Cartagena), dado que estos entes no cuentan con suficiente capital para implementar una política social hacia el exterior en forma permanente y sistemática. Los empleados deben ser considerados como parte activa de la organización y hacerles sentir que si la organización crece ellos igualmente lo harán. Es impensable considerar un enfoque socialmente responsable donde las condiciones de los empleados sean inadecuadas.

\subsection{Clientes}

Como se comentó anteriormente, los clientes conforman, junto a los empleados, los stakeholders a quienes se dirigirá principalmente la política social. Su importancia es evidente. Una gestión social que se oriente a la satisfacción de las necesidades personales y morales de los clientes, muy seguramente contribuirá con grandes beneficios para la organización. Es preciso traer a colación esa célebre frase que expresa que un "cliente satisfecho vuelve", y más aún, si siente que la organización que le prestó servicios está contribuyendo a mejorar sistemáticamente las condiciones sociales de sus grupos de interés.

\subsection{Proveedores}

$\mathrm{Si}$ bien es cierto que los proveedores no son representativos en número y en el volumen de las operaciones con respecto a las organizaciones del sector objeto de estudio, esta condición no constituye limitantes para no realizar una gestión eficiente frente a estos stakeholders. Se precisa elaborar un listado donde se detallen los principales proveedores con las metas a alcanzar fundadas en una gestión social responsable.

\subsection{Comunidad de Influencia}

La comunidad de influencia representa todos los grupos que se ven afectados por las decisiones que se originan desde los hoteles, y que se encuentran en los alrededores de sus instalaciones. En consecuencia, se deben identificar las principales organizaciones, instituciones o grupos de personas que cumplan con estas características, donde se precisen las metas que se desean alcanzar en una gestión social responsable con estos grupos, de acuerdo a las peculiaridades que surjan del análisis de cada uno.

\subsubsection{Diagnóstico}

Después de la identificación de los grupos de interés, se hace necesaria la realización de un diagnostico que se constituirá en el punto de partida para diseñar las estrategias y acciones socioambientales. Realizar estas acciones permitirá conocer de primera mano la realidad de las organizaciones para a partir de allí realizar una correcta planeación estratégica que oriente los procesos a seguir en un enfoque de gestión social responsable. El diagnostico pretende la búsqueda de información objetiva que permita saber con certeza cómo está cada organización con respecto al enfoque de responsabilidad social empresarial. Entre los puntos más sobresalientes que debe incluir el diagnostico están: determinar el grado de conocimiento que se tiene sobre la temática, principalmente los gerentes, 
que serán las personas bajo cuya responsabilidad se adoptará una gestión social; un análisis de las acciones realizadas en materia ambiental, principalmente de actividades que busquen la preservación del medio y aquellas que pretendan la medición de los impactos causados por las operaciones organizacionales; las consideraciones de empleados, clientes, proveedores y comunidad de influencia sobre la gestión realizada en materia social y ambiental, entre las que se destacan la incorporación de prácticas basadas en valores como el respeto y la tolerancia para el establecimiento de las relaciones (clima laboral, satisfacción de clientes y relaciones comerciales sanas y estables).

Es necesario que desde la gerencia se incentive la participación de todos los stakeholders en las actividades que implican el diagnostico, establecer mecanismos para una participación eficaz y en igualdad de condiciones para todos. Igualmente, incentivar la objetividad en todos los procesos y la determinación clara y expresa por parte de la gerencia de que no habrá recriminaciones ni consecuencias negativas por las consideraciones de cada grupo.

El diagnostico pretender identificar las fortalezas y debilidades que presenta cada organización en un momento dado, al igual que reflejar las oportunidades de mejora, las áreas críticas, y los requerimientos de las partes interesadas, que servirán para el diseño de las estrategias socioambientales.

\subsubsection{Diseño de estrategias socioambienta- les (Plan de Acción)}

Las estrategias que se implementarán dentro de una gestión socialmente responsable se constituirán como un Plan de Acción, en donde se especifiquen los lineamientos a seguir, en forma clara y concreta, entendible para todos en la organización. Este plan de acción se estructura de la siguiente manera:

\subsection{Principios y valores del Sistema}

Las estrategias sociales y ambientales que emanan del presente sistema se fundamentan en los principios que guían una gestión responsable, acorde con lineamientos éticos y morales presentes en la sociedad colombiana. Ésta, podría caracterizarse como tradicionalista, cimentada axiológicamente en principios de la fe cristiana producto del dominio de la Iglesia Católica desde inicios de la conquista, incluso en nuestros días. El primer paso en el diseño de estas estrategias será proclamar los principios éticos y morales que sustentarán todas las actividades y programas a implementar. Cada organización tendrá la absoluta libertad para acoger los principios que bien crea son fundamentales dentro de su gestión, y así dar cumplimiento a los objetivos y metas trazadas. Los principios y valores proporcionan identidad a los miembros de la organización, reflejan la cultura organizacional, y el ser humano que se quiere tener en el ente. Asimismo, clarifican el pensamiento y las acciones para mostrar transparencia en toda la organización.

\subsection{Restructuración de la misión, vi- sión y metas institucionales}

La misión, visión y metas institucionales sintetizan la política que orienta todos los procesos organizacionales. La misión incorpora el objeto social de cada entidad, su razón de ser y la forma como lo hace. Se constituye en el propósito de la empresa u organización. Proyecta los objetivos de la organización con base en las peculiaridades de cada una. Por su parte, la visión proyecta una imagen del futuro deseable de una organización. Es integradora y positiva, alienta a ser mejor cada día. Se enuncia clara y explícitamente argumentando hacia donde se dirige la empresa en el largo plazo, teniendo presente lineamientos como los valores institucionales y el personal con el que se cuenta. Finalmente, las metas institucionales representan los planes para alcanzar en el desarrollo de la misión y la visión. Al igual que la visión, las metas institucionales muestran una ruta de acción acorde con la política institucional.

Se propone un cambio en la filosofía institucional que integre un enfoque socialmente responsable en la misión, visión, metas, principios y valores institucionales. Una gestión social responsable precisa una armonización de la misión, visión y metas institucionales con los valores que identifican a la organización, al mismo tiempo que exige una incorporación de compromisos sociales y medioambientales que 
demuestren la intención de gestionar sus procesos con base a un enfoque de responsabilidad social. En otras palabras, se hace necesaria la incorporación de consideraciones socioambientales en la exposición de estos elementos.

\subsection{Políticas de gestión social}

Estas se constituyen en una enunciación clara, concreta y alcanzable de las intenciones de asumir compromisos en beneficio de la sociedad y el medio ambiente. Deben estar en armonía con los lineamientos axiológicos de cada organización y responder a las necesidades diagnosticadas de los stakeholders en la fase inicial. Este paso incluye la estructuración de una política social, una ambiental, una de buenas relaciones, una de precios y una de pagos.

La política social se constituye en una filosofía que se enmarca dentro de los principios de la organización, que emana de la responsabilidad que se tiene con toda la sociedad para propiciar el desarrollo y el bienestar social. Su principal intención es integrar en todos los procesos organizacionales el compromiso que se tiene como empresa generadora de ingresos, tanto para propietarios como para empleados y proveedores, pero con sentido humano, y el involucramiento de los empleados en los ámbitos organizativos, basados en el dialogo y la concertación.

La política ambiental recopila los compromisos en la materia, que redundan principalmente en la expresa intención de procurar mecanismo de prevención de impactos negativos en el medio, así como la implementación de acciones de preservación del ambiente.

La política de buenas relaciones se extiende a todos los stakeholders y se cimenta en los valores organizacionales, esencialmente el respeto y la tolerancia. Consiste en una enunciación donde se establecen lineamientos para el mantenimiento de relaciones idóneas con todos en la organización, entre los cuales se destacan la comunicación efectiva y el diálogo.

La política de precios y la de pagos sintetizan el compromiso con clientes y proveedores respectivamente, ofreciéndole a los primeros seguridad y confianza al momento de la liquidación y/o facturación de los servicios que ofrece el hotel, y a los segundos certeza para el cumplimiento de las obligaciones que surjan en la compra de productos o servicios.

\subsection{Programas de capacitación}

Los programas de capacitación constituyen una de las estrategias más sobresalientes dentro de una gestión socialmente responsable. Es bien sabido que los empleados son el grupo de interés más sensible y cercano de la empresa, y es en estos que debe originarse la gestión social, con el compromiso de que se sientan conformes con su trabajo, que reciban el apoyo de la organización donde laboran, y sobre todo, que sientan que si la empresa está en crecimiento ellos igualmente puedan mejorar sus condiciones. Los programas de capacitación deben diseñarse con base en el diagnóstico realizado, haciendo énfasis en las deficiencias de información que puedan presentar los empleados, y temas de interés general, como salud sexual y reproductiva, que encierra mitos y tabúes a pesar de los adelantos a los que se ha llegado en la forma $y$ métodos de educación sexual.

Las primeras capacitaciones deben incluir la socialización efectiva de los objetivos del Sistema de Gestión en Responsabilidad Social Empresarial para Pequeños hoteles de la Ciudad de Cartagena, así como los beneficios y ventajas de implementar prácticas y principios socialmente responsables, y su importancia como estrategia competitiva y diferenciadora. Esta constituye la fase de armonización.

Asimismo, se deben realizar capacitaciones profesionalizantes, que consisten en la instrucción del personal de la organización en habilidades, conocimientos, actitudes y destrezas propias de su cargo o puesto de trabajo. Esto genera un mejor desempeño de su trabajo, además de mantenerlos permanentemente actualizados con relación a las innovaciones y cambios científicos y tecnológicos que producen ventajas competitivas frente a los competidores. Otros temas de interés general son los siguientes: salud ocupacional, prevención de riesgos y enfermedades profesionales, finanzas personales, idiomas, etc. 
Si es posible, a las capacitaciones pueden asistir personas de la comunidad de influencia, clientes y proveedores. Para lograr una recepción efectiva de la información, se deben crear los ambientes propicios para ello y disponer del tiempo necesario, y que sean permanentes, con involucramiento de los directivos o propietarios. Se cuenta con la disposición de instituciones como la Cámara de Comercio de Cartagena, la Universidad de Cartagena y el SENA para la realización de las capacitaciones profesionalizantes.

\subsubsection{Documentación de la etapa}

La documentación de cada etapa consiste en la elaboración de un informe que muestre evidencias de la gestión, logros alcanzados, dificultades presentadas en cada proceso o actividad y la forma como se hizo frente a dicha situación. Igualmente, se deben incluir los hallazgos que se consideren interesantes y hechos sobresalientes. La intención es tener la suficiente información y documentos para poder elaborar un informe final completo y ajustado a la realidad (etapa final del sistema).

\subsubsection{Implementación}

La implementación se convierte en la etapa donde lo planeado se ejecuta, donde el compromiso expresado se hace evidente. Se materializan los objetivos propuestos en materia de responsabilidad social empresarial. Aquí la gestión social adquiere una real dimensión, dado que pasa del papel (planeación) a la realidad. Una correcta implementación depende de una planeación eficiente. Por ello, es preciso abarcar todos los lineamientos propuestos en la etapa inicial para avanzar con paso firme hacia la ejecución de las actividades propuestas en la materia. El responsable directo de todos los procesos que implican una gestión social responsable es el administrador o gerente de cada hotel, dado que este al conocer todas las esferas organizacionales se convierte en la persona más idónea y capacitada para asumir tal compromiso. La implementación se desarrolla siguiendo las fases de armonización, de desarrollo de estrategias socioambientales y respectiva documentación de cada proceso.

\subsubsection{Armonización}

La armonización constituye la fase donde se realizarán capacitaciones sistemáticas sobre responsabilidad social empresarial y sistemas de gestión social y ambiental. Igualmente, es precisa la divulgación de los objetivos de la investigación a realizar (diagnostico), las metas que se esperan alcanzar, y sobre todo, los beneficios que se obtendrán tras una implementación de un enfoque de gestión social responsable. La armonización bien podría convertirse en un programa de capacitaciones a todos los stakeholders, principalmente los empleados que son los que tendrían la disponibilidad para asistir a las sesiones de capacitación.

\subsubsection{Desarrollo de estrategias socioam- bientales}

Esta fase comprende la implementación de todas las actividades planeados en la etapa inicial. Se estructura en diversos procesos que se materializan desde la dirección de cada hotel, los cuales se detallan a continuación:

\subsection{Gestión Social}

Estas actividades procuran dar bienestar y comodidad a los empleados y clientes, principalmente, y a los proveedores y personas de la comunidad de influencia del ente. Deben incluir la construcción de las políticas de gestión social, las cuales resumen el compromiso por parte del hotel para afianzar la responsabilidad social empresarial.

Como se enunció anteriormente, los valores y principios dan sentido a las actividades programadas, y orientan las acciones bajo un sentido humanístico. El respeto, la dignidad, el compromiso, la tolerancia y la solidaridad son valores y principios esenciales en el Sistema de Gestión en Responsabilidad Social Empresarial para Pequeños hoteles de la Ciudad de Cartagena.

La gestión social dirigida a empleados tiene que ver con la disposición absoluta de las directivas del hotel para cumplir las obligaciones contractuales contempladas en la legislación laboral colombiana, y brindar apoyo, bienestar y seguridad a los mismos. Con respecto a los clientes es importante generar una relación fundada en la confianza mutua y la lealtad, que se refleje en 
una política de precios donde éstos sean acordes con la realidad del hotel y de la ciudad, y brindar comodidades y confort. Asimismo, se deben procurar esfuerzos para afianzar alianzas con agencias de viaje, y otros hoteles para fortalecerse como sector en crecimiento.

Las acciones dirigidas a la comunidad deben ser concretas, alcanzables, y puedan ser medidas para conocer sus impactos. Además deben centrarse en los lineamientos éticos que sustentan el sistema de gestión en responsabilidad social empresarial. Para relaciones con la comunidad de influencia los canales de comunicación deben ser efectivos, en este caso son indispensables, dado que es con este grupo de interés con el cual se tiene menor oportunidad de interacción. Hacia los proveedores el compromiso radica en el mantenimiento de buenas relaciones y el pago oportuno de las obligaciones contraídas.

\subsection{Gestión de la Innovación y el Em- prendimiento}

Dentro de este proceso se debe estimular a los empleados a afrontar nuevos retos, a mejorar procesos y crear ideas emprendedoras e innovadoras. Para ello se pretende incitarlos a adoptar una actitud hacia la identificación de oportunidades y el desarrollo de nuevos productos, servicios y modelos de negocio que generen valor para la organización. Todos estos procesos están relacionados con el crecimiento económico, el tamaño y la dinámica de los mercados, la estrategia de las empresas, la identificación de necesidades latentes en los consumidores, la generación y adaptación de conocimiento y tecnología, la capacidad de los individuos para crear y actuar en grupos interdisciplinarios y la gestión de procesos para mantener un flujo de nuevos productos y servicios.

\subsection{Gestión del talento humano}

Estas actividades se desarrollan en distintas etapas que surgen de la administración del capital humano de la organización, desde el reclutamiento, selección, contratación, inducción, capacitación y entrenamiento, remuneración, promoción, programas de bienestar social, y terminación de contratos. Los programas de capacitación deben ser permanentes, e incluso pueden ser concertados con los empleados. La capacitación en temas de RSE, y cuidado, preservación y prevención de impactos negativos en el medio ambiente deben ser los ejes del sistema. Capacitarse para conocer verdaderamente la magnitud de las acciones humanas y prever posibles consecuencias negativas para el medio, la sociedad y la empresa misma es la clave para el éxito de una gestión social responsable. La gestión del talento humano se sintetiza en el hecho que los empleados se sientan a gusto en la organización, que sientan que se está con ellos y que se está trabajando para darles mayor bienestar y comodidad. Asimismo, es importante que los empleados reciban capacitaciones que les permitan prestar los primeros auxilios a cualquier persona en la organización en caso de accidentes, mientras se busca ayuda profesional, principalmente en hoteles del centro histórico donde no se encuentran ubicadas instituciones de salud y el acceso de ambulancias podría llegar a ser dificultoso.

\subsection{Gestión Ambiental}

Permite la creación de una cultura organizacional basada en políticas y prácticas amigables con el medio ambiente. Estas actividades contemplan la creación de un Sistema de Gestión Ambiental Simplificado que permita el manejo de residuos sólidos, propenda por el reciclaje, el uso racional de recursos como el agua y la energía eléctrica, la utilización de productos de limpieza no contaminantes (detergentes y desinfectantes biodegradables o blandos). Para complementar el mismo, se hace imperante el contacto con asociaciones de recicladores con quienes se puede establecer alianzas para la recolección de materiales reciclables, al tiempo que se generan mayores oportunidades de ingresos para esa población.

\subsection{Gestión del Conocimiento}

Hace referencia a la optimización en el uso que se hace del conocimiento generado dentro de la organización. Comprende actividades programadas para crear las condiciones necesarias para hacer uso, compartir y desarrollar los conocimientos de las personas que laboran en una entidad.

El ser humano es capaz de sistematizar procesos en su mente al realizar actividades cotidia- 
nas y permanentes, que le permiten especializarse en ellas a tal punto que se vuelven expertos, logrando así utilizar su tiempo y destrezas de forma eficiente, al mismo tiempo que puede convertir estas habilidades en ventajas diferenciadoras y que den valor agregado a su trabajo. Cuando se ha llegado a este punto, la gestión del conocimiento permite direccionar las actividades hacia escenarios donde se pueda documentar y sistematizar la información generada en estos procesos.

\subsection{Gestión del Cambio}

Estas actividades se desarrollan para cambiar las costumbres de los empleados, e incluso de los mismos directivos, hacia hábitos y formas de vidas saludables, ambientales y con sentido de pertenencia por la organización y el entorno que los rodea. Además, en este punto se deben prever los posibles cambios y/o ajustes necesarios para armonizar el alcance y objetivos del sistema con las circunstancias imprevistas que puedan surgir en el desarrollo de las actividades.

\subsection{Gestión Gubernamental}

Encierra principalmente funciones relacionadas con estar en contacto con las autoridades locales y entidades gubernamentales que apoyan el sector turismo, como el Servicio Nacional de Aprendizaje - SENA, la Cámara de Comercio, la Corporación Turismo Cartagena de Indias, la Asociación Hotelera y Turística de Colombia COTELCO, entre otros. Asimismo, estar pendiente de todos los programas gubernamentales que traen beneficios e incentivos al sector hotelero, particularmente de Proexport, del Fondo de Promoción Turística y del Ministerio de Comercio, Industria y Turismo; al mismo tiempo organismos como el Banco de Comercio Exterior de Colombia - BANCOLDEX, ofrecen facilidades de crédito para financiar actividades turísticas. El sistema debe contribuir a la generación de información sobre temas del sector turismo para las autoridades locales, departamentales y nacionales, que puedan alimentar un sistema de información en turismo sostenible.

\subsubsection{Documentación de la etapa}

Al igual que en la etapa de planeación, es preciso documentar todos los procesos y actividades para poder incluir cada detalle en los respecti- vos informes, que sin duda se convertirán en materia prima para procedimientos de retroalimentación. Los borradores sobre cada actividad desarrollada deben archivarse para poder redactar un informe sobre el avance de las actividades programadas, logros alcanzados, dificultades presentadas en cada proceso o actividad y la forma como se hizo frente a dicha situación, así como los hallazgos y hechos que se consideren interesantes y sobresalientes.

\subsubsection{Aseguramiento}

El aseguramiento constituye una etapa clave para identificar debilidades y deficiencias en los procesos ejecutados, y las diferencias con las actividades planeadas. Busca realizar los ajustes necesarios para que la gestión social alcance su mayor dinamismo dentro de cada organización. Se pretende establecer un pare en el desarrollo de las estrategias sociales, para analizar todas las actividades y procesos ejecutados, detallar las características de cada uno, y en caso de presentarse deficiencias o errores, subsanar estos aspectos. En este punto la mejora continua representa un elemento clave para posicionar las estrategias sociales en toda la organización. Por ello, una actitud de mejoramiento continuo es indispensable para lograr que la gestión social se convierta en una estrategia clave que beneficie a todo el ente. El aseguramiento se estructura en las fases de verificación, ajustes, corrección y la documentación de cada una.

\subsubsection{Verificación y control de cumplimiento}

La verificación permite medir el grado de cumplimiento de cada una de las fases del sistema, de tal suerte que aquellas que necesiten ajustes, tengan un mayor monitoreo por parte del responsable del sistema. La verificación debe ser autónoma, y desarrollarse bajos los planteamientos y rigurosidad de una auditoría. Debe ser ejecutada bajo la dirección y responsabilidad del gerente y/o administrador. Se pueden buscar profesionales independientes para realizar una verificación de la misma, para dar mayor seguridad a los controles establecidos. La verificación permanente de la gestión social 
realizada permitirá sin duda detectar las desviaciones que pudieran llegar a presentarse.

La gestión en responsabilidad social empresarial debe ser medida con indicadores propios construidos atendiendo las características de las organizaciones objeto de estudio. Estos pueden ser cuantitativos o cualitativos, y permitirán medir la gestión realizada, para verificar su cumplimiento y así determinar los ajustes del caso. Los indicadores se construyen por cada estrategia social implementada. Los indicadores deben permitir la medición de los impactos que la gestión social tiene sobre los grupos de interés, para así poder saber con certeza si las acciones implementadas en materia social contribuyen al desarrollo y bienestar de estos.

\subsubsection{Ajustes y corrección}

Los indicadores construidos al ser aplicados mostrarán si realmente la gestión realizada ha alcanzado el nivel deseado, el cual debe ser detallado con cada indicador de tal manera que permita hacer el seguimiento y la medición de los procesos, y dar muestras del avance del sistema. En caso de que existan procesos que los indicadores muestren con deficiencias y/o debilidades, se debe proceder a hacer los correctivos y ajustes necesarios para alcanzar los objetivos y metas proyectadas.

Los ajustes deben ser aplicados en el momento oportuno para que puedan surtir el efecto deseado. Los ajustes implican procedimientos para encauzar la gestión por el rumbo correcto. La corrección se aplica cuando la situación está totalmente fuera de lo previsto. Por ello, como primera medida se deben hacer ajustes antes que correcciones.

\subsubsection{Documentación de la etapa}

Como en las etapas predecesoras, la documentación representa la forma más confiable de dar seguridad a la última etapa del sistema. Si cada fase se documenta correctamente, realizando los informes necesarios y tomando las evidencias suficientes, se podrá redactar un informe final de toda la implementación del sistema completo y con la facilidad requerida.

\subsubsection{Comunicación}

Esta etapa se constituye en la estrategia más importante para poder informar a toda la sociedad los adelantos en materia de responsabilidad social empresarial. En el diagnóstico del sector objeto de estudio se detectó una total ausencia de informes y documentación de los procesos $\mathrm{y}$ actividades que se realizan en la temática. Por ello la importancia que este sistema pretende dar a la comunicación de la gestión social responsable. Las empresas pudieran estar realizando acciones concretas en tema socioambientales, pero en ausencia de organización, control y documentación de dichas acciones, estas quedarían fácilmente en el olvido y sin la posibilidad de servir como referentes para futuras actividades. Esta etapa implica la gestión de la información, la cual se ha estado realizando desde el comienzo del sistema, con la documentación de cada etapa.

En las opiniones y comentarios de los stakeholders, está una fuente de información muy valiosa, dado que estos observan la gestión social realizada por la organización desde otro ángulo, lo que puede ayudar a formarse una idea más holística de dicha gestión. En consecuencia, es preciso elaborar el informe sobre la gestión social de la empresa en un dialogo abierto con los grupo de interés como principales agentes evaluadores de las prácticas en RSE implementadas por la empresa. La etapa de comunicación se estructura en la estrategia y plan de comunicación y la elaboración de los informes socioambientales.

\subsubsection{Estrategia y plan de comunicación}

La estrategia y plan de comunicación tienen como principal objetivo hacer visible la organización, sobre todo la gestión que se realiza en materia social y ambiental, la cual sin duda busca promover el desarrollo y la contribución a la solución de problemas en la sociedad, y así debe expresarse en los informes realizados. El compromiso adquirido incluye la socialización de las estrategias implementadas a todos los grupos de interés, mostrar el trabajo realizado y tal vez inspirar a otras organizaciones a seguir el rumbo de la gestión social responsable como herramienta estratégica. En otras palabras, la 
comunicación de la gestión social se convierte en una estrategia para dar reputación y posicionamiento a cada organización.

El plan de comunicación implica una proyección de la gestión social responsable hacia el exterior. Lo importante es llegar al público requerido, a través de la Internet (páginas web propias, redes sociales como Facebook, Twitter o Youtube, portales empresariales y/o académicos), boletines o revistas especializadas sobre el sector hotelero o sobre responsabilidad social empresarial. Asimismo, es preciso programar reuniones con los grupos de interés donde se socialicen los resultados de la gestión social responsable realizada.

\subsubsection{Reportes socioambientales}

Los reportes socioambientales deben integrarse a los informes de carácter financieros que emita cada organización, de tal suerte que se evidencie una gestión integral y sólida, con transparencia, como una verdadera rendición de cuentas, que al mismo tiempo genere confianza y aceptación en la sociedad. Además, con estas prácticas se genera reputación y posicionamiento en la sociedad. Estos reportes se constituyen como un documento donde se informe de manera formal, concreta y entendible las actividades que se implementaron dentro del Sistema de Gestión en Responsabilidad Social Empresarial para Pequeños hoteles de la Ciudad de Cartagena, los alcances de las mismas, los beneficiarios de cada una y los impactos causados a los grupos de interés. Igualmente, los resultados de la aplicación de los indicadores y su comparación con las metas propuestas. Los informes deben incluir archivos gráficos como fotografías, videos, reseñas, los cuales pueden ser cargados en páginas web propias o redes sociales.

\section{CONCLUSIONES}

La responsabilidad social empresarial se constituye en un imperativo para los enfoques modernos de gestión organizacional, que buscan la incorporación de elementos sociales en las decisiones gerenciales de alto impacto y su contribución al desarrollo de los grupos sociales que se ven afectados por estas, principalmente los empleados.
El actuar empresarial es posible ampararlo en una perspectiva social que se involucre totalmente en todos los niveles y elementos de las empresas, que fortalezca la toma de decisiones estratégicas y contribuya a su posicionamiento y buena reputación, al tiempo que permita mayores índices de productividad y desarrollo organizacional. La gestión social incorporada a la gestión financiera y operativa fortalece las estructuras del ente, condiciona las acciones a patrones de conducta sustentados en principios éticos de aceptación general, y se constituye en un valor agregado que genera beneficios de estatus y diferenciación de este sector empresarial, el cual contribuye a la creación de gran cantidad de empleos directos e indirectos y es considerado como una de las apuestas productivas de Bolívar.

Los resultados evidencian que una visión socialmente responsable es aplicable a cualquier organización, independientemente de su tamaño o sector económico. Aplicar preceptos socialmente responsables humaniza los procesos empresariales y genera valor agregado a las organizaciones que los interiorizan. Ser eficientes en todos los procesos, respetar el medio ambiente y ser partícipe de las problemáticas de los empleados, es el comienzo del camino hacia la gestión social sustentable. Esto no genera gastos adicionales, más bien requiere una inversión de tiempo y esfuerzo por parte de empresario y/o gerente. La gestión social define un enfoque de conciencia, más no altruista.

Los resultados de la caracterización evidencian prácticas cercanas a la responsabilidad social empresarial, pero carentes de un referente teórico que las complemente y fortalezca.

En general, se evidencia la disposición de los empresarios del sector para afrontar nuevas posibilidades de gestión, para estructurar mecanismos y herramientas modernas de organización que materialicen el compromiso de contribuir al desarrollo de su sociedad, y anticipación a la ocurrencia de problemáticas que pudieran perturbar el buen funcionamiento de la entidad.

Por ello, la RSE debe convertirse en una estrategia de competitividad que genere beneficios 
y valor agregado a la organización, de lo contrario perdería su funcionalidad y su atractivo entre los empresarios.

En razón de estos argumentos, el Sistema de Gestión en Responsabilidad Social Empresarial para Pequeños hoteles de la Ciudad de Cartagena se constituye en una poderosa herramienta que orienta el accionar empresarial ha- cia nuevos horizontes de gestión organizacional, donde los intereses netamente financieros se conjugan con los sociales y ambientales para generar una cultura de compromiso y respeto con las necesidades de los grupos de intereses, las cuales van desde el imperativo de bienestar y desarrollo hasta el deseo de información (informes sociales, rendición de cuentas, transparencia empresarial, etc.).

\section{BIBLIOGRAFÍA}

CASTELlanOS, J. (2003). Pymes Innovadoras: Cambios de Estrategias e Instrumentos. Revista Escuela de Administración de Negocios, No. 47, pp.10-33.

SIERRA, J. y LONDOÑO, D. (2008). RES y MiPymes, alternativas para apoyar su desarrollo en Colombia. Contaduría Universidad de Antioquia, 52, 13-44.

DEPARTAMENTO NACIONAL DE PLANEACIÓN Y MINISTERIO DE COMERCIO, INDUSTRIA Y TURISMO. (2007). Documento CONPES 3484. Política nacional para la transformación productiva y la promoción de las micro, pequeñas y medianas empresas: Un esfuerzo público-privado. Bogotá. Recuperado de http://www.acopi.org.co/dynamicdata/ asset/Conpes\%203484.pdf

CARBAL, A. (2009). Responsabilidad Social Empresarial y Contabilidad: Apuntes Críticos. Universidad Libre Cartagena. Recuperado de http://unilibrectg.edu.co/PDF/RESPONSABILIDAD_SOCIAL.pdf.

SÁNCHEZ, I., JULIAO, J. y ZULUAGA, J. (2013). La relación entre las redes externas de trabajo y el desempeño innovador de las pymes colombianas: un análisis del rol moderador del ambiente industrial. Estudios Gerenciales. 29.128 p339. MONTOYA, A., MONTOYA, I. y CASTELLANOS, O. (2010). Situación de la competitividad de las Pyme en Colombia: elementos actuales y retos. Agronomía Colombiana, 28(1), 107-117

GARZÓN D., (2005). Programa sobre operaciones colectivas entre empresas. En: Seminario Iboamericano sobre Integración Empresarial y Cooperativa: cooperar y exportar para ganar. Convenio BID-ATN/ME 7856 CO. Recuperado de www. iberpymeonline.org/venezuela130705/ricardogarzon.pdf

GIRALDO R., (2007). Estadísticas de la microempresa en Colombia: análisis comparativo 1990-2005. Corporación para el Desarrollo de las Microempresas, Bogotá.

SUPERINTENDENCIA DE SOCIEDADES, (2005). Las MiPymes en Colombia años 2002 - 2004. Recuperado de http:// sirem.supersociedades.gov.co/SIREM/files/estudios/PYMES_1.pdf

ZEVALlOS, E. (2006). Obstáculos al desarrollo de las Pequeñas y Medianas Empresas en América Latina. Recuperado de http://www.esan.edu.pe/paginas/publicaciones/cuadernos/20/Zevallos.pdf

VELÁSQUEZ, F. (2004). La estrategia, la estructura y las formas de asociación: fuentes de ventaja competitiva para las Pymes Colombianas. Estudios Gerenciales, Vol.20, No.93, p.73-97.

SÁNCHEZ, J., OSORIO, J. y BAENA, E. (2007). Algunas aproximaciones al problema de financiamiento de las Pymes en Colombia. Revista Scientia et Technica Universidad Tecnológica de Pereira, Año XIII, No 34, Pág. 321-324. 
RODRÍGUEZ, A. (2003). La realidad de la pyme colombiana - Desafio para el desarrollo. Bogotá: FUNDES. Recuperado de http://www.fundes.org/uploaded/content/publicacione/1241969270.pdf

MELO, A. 2003. Colombia: los problemas de competitividad de un país en conflicto. Serie de estudios de competitividad. Documento de trabajo \# C-103. Recuperado de http://www.iadb.org/res/publications/pubfiles/pubc-103.pdf

PUYANA, D. (2010). La Problemática De Las Pymes en Colombia: Internacionalizarse o Morir. Recuperado de http:// www.usergioarboleda.edu.co/pymes/noticia1.htm

MENDOZA, G. (2012). Análisis del Sector Turismo en Cartagena como Elemento de Internacionalización de la Ciudad. Trabajo de grado publicado, Programa de Administración de Negocios Internacionales, Universidad del Rosario, Bogotá D.C.

QUINTERO, P., BERNAL, C. y LÓPEZ, H. (2005). La Competitividad Turística de Cartagena de Indias: análisis del destino y posicionamiento en el mercado. Serie de Estudios sobre Competitividad de Cartagena, No. 4. Observatorio del Caribe Colombiano y Cámara de Comercio de Cartagena.

ALCALDÍA MAYOR DE CARTAGENA DE INDIAS, CORPORACIÓN TURISMO CARTAGENA DE INDIAS, INSTITUTO DE PATRIMONIO Y CULTURA, CORPORACIÓN CARTAGENA Y PROGRAMA DE LAS NACIONES UNIDAS PARA EL DESARROLLO, (2004). “Cartagena de Indias es nuestro Patrimonio” Plan Sectorial de Turismo de Cartagena de Indias.

SIERRA, G., MARRUGO, D. y QUEJADA, R. (2004). La actividad del turismo en Cartagena de Indias, Cartagena: Editorial Universitaria.

MALDONADO, C. (2006). Turismo y comunidades indígenas: Impactos, pautas para autoevaluación y códigos de conducta. En: Serie Red de Turismo Sostenible Comunitario para América Latina (REDTURS) Documento de trabajo núm. 79. Programa de Desarrollo de Pequeñas Empresas. Departamento de creación de empleos y desarrollo de la empresa. Recuperado de http://www.redturs.org/inicio/docu/turismo_conducta.pdf

RAMOS, J. (2012). Cartagena de Indias (Colombia): la transición de sol y playa a destino cultural como estrategia de innovación, renovación y reestructuración del destino turístico en su ciclo de vida. En: Renovación y reestructuración de destinos turísticos en áreas costeras. Publicaciones Universidad de Valencia.

OTERO, J. (2008). La formación de actividades ilegales en la ciudad de Cartagena a partir de la concentración de la gestión Distrital en el sector del turismo. Análisis del periodo: 2002-2006. (Tesis de pregrado). Universidad del Rosario, Bogotá D.C.

COMISIÓN REGIONAL DE COMPETITIVIDAD DE CARTAGENA Y BOLÍVAR, (2010). Plan Regional de Competitividad Cartagena y Bolivar 2008-2032. Recuperado de http://www.cccartagena.org.co/temp_downloads/plan-regional-decompetitividad-cartagena-y-bolivar-2008-2032.pdf

OGALLA, F. (2006). La integración de la Responsabilidad Social en el sistema de gestión de la empresa. Recuperado de http://www.cyta.com.ar/biblioteca/bddoc/bdlibros/rse/334_as_integracion_rse.pdf

GÓMEZ, H. (2009). Desarrollar destrezas para la competitividad de Colombia. Altablero No. 48. Ministerio de Educación Nacional. Recuperado de http://www.mineducacion.gov.co/1621/article-183961.html.

PINTO, L., BECERRA, L. y GÓMEZ, L. (2012). Carencias en los sistemas de gestión del conocimiento: una revisión bibliográfica. Recuperado de http://eprints.rclis.org/17477/1/definitivo.pdf 
SOLANA, P. y PÉREZ, D. (2008). Estrategia empresarial y tecnologías de la información en la gestión del conocimiento técnico-documental. Estudio del caso Nuclenor. Recuperado de http://www.elprofesionaldelainformacion.com/contenidos/2008/septiembre/02.pdf

INSTITUTO ETHOS DE EMPRESAS Y RESPONSABILIDADE SOCIAL Y SERVICO BRASILEIRO DE APÓIOÁS MICRO E PEQUEÑAS EMPRESAS (SEBRAE), (2007). Indicadores Ethos-Sebrae de Responsabilidad Social Empresarial para Micro y Pequeñas Empresas. Recuperado de http://www.ethos.org.br/_Uniethos/documents/IndicadoresEthosSebraeEspanhol.pdf

ICONTEC. (2008). Guía Técnica Colombiana de Responsabilidad Social-GTC 180.

VELOZ, E. (2008). ISO 26000 y Derechos Laborales. Reflexiones y perspectivas desde las organizaciones sindicales y ciudadanas. Recuperado de http://www.mapeo-rse.info/sites/default/files/ISO_26000_y_derechos_laborales_reflexiones.pdf GLOBAL REPORTING INICIATIVE - GRI, (2006). Guía para la elaboración de Memorias de Sostenibilidad. Versión 3.0. Recuperado de http:/www.globalreporting.org/NR/rdonlyres/54851C1D-A980-4910-82F1-0BDE4BFA6608/5426/ G3_SP_RG_Final_with_cover1.pdf

GUAJARDO, A. (2007). Modelo y herramientas de gestión de RSE. Facultad Economía y Negocios Universidad de Chile. Recuperado de http://es.scribd.com/doc/37966567/Herramientas-de-SISTEMA-GESTION-DE-RSE

DEL CASTILLO, M., BONNET, A., HENNESSEY, P., Quiroz, J. y Rizzo, S. (2012). Responsabilidad Social Empresarial: Prioridad de la Cultura Organizacional. En: Ingeniare, Universidad Libre Barranquilla. Año 7, No. 13, pp. 95-102

MOLINA, J. (2009). Balance y perspectiva de las normas de responsabilidad social - ISO 26000 y NMX-SAST-004-IMNC. $7^{\circ}$ Congreso Internacional de Responsabilidad Social - México D.F. Octubre de 2009. Recuperado de www.compite.org. $\mathrm{mx} /$.../Congreso\%20COMPITE\%20Jorge\%20Molina.ppt

MORA, C. (2005). La responsabilidad social empresarial: Razón de ser del Marketing como sistema de pensamiento organizacional. En: Visión Gerencial, Año 4, No. 2, Vol. 4, pp. 171-179

ARISTIMUÑO, M., VELÁSQUEZ, Y. y RODRÍGUEZ, C. (2010). La Responsabilidad Social Empresarial (RSE), como valor organizacional en la Pequeña y Mediana Industria (PYME). 8th Latin American and Caribbean Conference for Engineering and Technology, Arequipa, Perú, Junio 1-4. Recuperado de http://www.laccei.org/LACCEI2010-Peru/published/ EE031_Aristimuno.pdf

FONTALVO, T., y BOLÍVAR, M. (2010). El sistema de gestión de la responsabilidad social empresarial como una estrategia para la prevención de la contaminación y de los riesgos profesionales. En: Escenarios, Vol. 8, No. 2, pp. 13-20

VALVERDE, J. y BERMÚDEZ, J. (2012). Así se hace: Gestión de la responsabilidad social empresarial y casos de buenas prácticas. Universidad Nacional de Costa Rica. Recuperado de http://redunirse.org/nuevo/sites/default/files/asisehace_gestionrseybuenaspracticas.pdf 